\title{
Improved Simulations of the Planetary Nebula Luminosity Function
}

\author{
R. H. Méndez and T. Soffner \\ Munich University Observatory
}

We have developed a new procedure for the generation of a planetary nebula luminosity function (PNLF), improving on previous work (Méndez et al. 1993 A\&A 275, 534). The procedure is based, as before, on an exponential central star mass distribution, on $\mathrm{H}$ burning post-AGB evolutionary tracks, and on the avoidance of nebular models for the calculation of nebular fluxes. We have added new post-AGB evolutionary tracks (Blöcker 1995 A\&A 299, 755) and introduced the following improvements:

(1) the imperfect analytical representation of post-AGB evolutionary tracks has been replaced by an interpolation routine giving a better approximation;

(2) we have modified the distribution of the intensities of [O III] $\lambda 5007$ relative to $\mathrm{H} \beta$, so that it better imitates the observed distribution, which we have taken from data in the Strasbourg-ESO catalogue of Galactic PNs (Acker et al. 1992) and other sources;

(3) we have adjusted the absorbing factor $\mu$ along the white dwarf cooling tracks, so as to reproduce the observed PNLF of the Magellanic Clouds and M 31 at fainter magnitudes ( $\mu$ expresses the fraction of stellar ionizing luminosity absorbed by the nebula).

In this way we have produced a PNLF which is more consistent with observed PN properties. We use this randomly generated PNLF to:

(1) show as convincingly as possible that most PNs in any real population must leak stellar $\mathrm{H}$-ionizing photons;

(2) revise our estimates of the parameter $\mu_{\max }$, which now becomes much closer to unity. What is $\mu_{\max }$ ? Our procedure defines, for central stars on heating tracks and with temperatures above $40000 \mathrm{~K}$, a random distribution of $\mu$ from 0.05 up to a parameter $\mu_{\max }$. If $\mu_{\max }=1$, then a small percentage of the bright PNs with hotter central stars can have $\mu$ close to 1 .

(3) discuss the shape of the PNLF, hinting at the possible existence of a change of slope at $M(5007)=-2.3$.

(4) attempt estimates of maximum post-AGB final masses from fits to the PNLFs of the LMC and M 31. There is marginal evidence of a higher maximum final mass in the LMC, but this needs confirmation in other galaxies with recent star formation.

Several 8-m class telescopes will soon become available. This will lead to explosive progress in the field of extragalactic PNs. We expect the new PNLF simulations to be very useful for future PNLF distance determinations as well as for studies of the initial-to-final mass relation and related mass loss processes in luminous galaxies with and without recent star formation.

A paper with a full description of this work is in press (A\&A 1997). 